\title{
Use of mobile tablet devices and reduction in time to perioperative transesophageal echocardiography reporting: a historical cohort study
}

\author{
Utilisation de tablettes mobiles et raccourcissement du délai \\ d'envoi des comptes rendus d'échocardiographie \\ transosophagienne périopératoires: une étude de cohorte \\ historique
}

\author{
Brandi Bottiger, MD · Sharon McCartney, MD • Igor Akushevich, PhD • \\ Alina Nicoara, MD · Mamata Yanamadala, MBBS $\cdot$ Madhav Swaminathan, MD
}

Received: 19 August 2014/Accepted: 10 October 2014/Published online: 28 October 2014

(C) Canadian Anesthesiologists' Society 2014

\begin{abstract}
Purpose Timely communication of intraoperative transesophageal echocardiography (TEE) findings to the postoperative care team is critical to optimizing patient care. We compared the use of a personal computer $(P C)$ system with the use of a mobile tablet device (MTD) system
\end{abstract}

\begin{abstract}
Author contributions Brandi Bottiger and Sharon McCartney, cofirst authors, were instrumental in the concept and design of the study and acquiring, analyzing, and interpreting data. Additionally, Brandi Bottiger serves as corresponding author. Igor Akushevich provided statistical support and analysis from the conception and design of the study, provided input regarding data collection, analyzed and interpreted the data, and drafted and revised the manuscript into this final version. Alina Nicoara and Mamata Yanamadala contributed to the concept and design of the study and the analysis and interpretation of data. Madhav Swaminathan, as senior author, was involved in providing feedback and insight regarding the conception and design of the study and contributed to data acquisition, analysis and interpretation. Brandi Bottiger, Sharon McCartney, Alina Nicoara, Mamata Yanamadala, and Madhav Swaminathan were involved in drafting and revising the article for important intellectual content.
\end{abstract}

Bottiger and McCartney contributed equally as first authors.

B. Bottiger, MD $(\bowtie) \cdot S$. McCartney, MD · I. Akushevich, PhD .

A. Nicoara, MD · M. Swaminathan, MD

Division of Cardiothoracic Anesthesiology and Critical Care Medicine, Department of Anesthesiology, Duke University Medical Center, Box 3094 DUMC, Durham, NC 27710, USA e-mail: brandi.bottiger@dm.duke.edu

M. Yanamadala, MBBS

Division of Geriatrics, Department of Medicine, Duke University Medical Center, Durham, NC 27710, USA for point-of-care TEE data entry and hypothesized that the MTD-based system would reduce the time to preliminary TEE reporting and decrease the incidence of delinquent reporting by $50 \%$.

Methods In this historical cohort study, we reviewed 508 perioperative TEE reports entered by cardiothoracic anesthesia fellows. Reports were grouped based on whether data were entered on a PC (PC group) or a MTD (MTD group). Time to TEE reporting was defined as the time from the patient leaving the operating room to the time the TEE report was generated. Delinquent reports were defined as those generated $>24 \mathrm{hr}$ after the initial exam. Time to TEE reporting and incidence of delinquent reports were compared between the two groups.

Results Mean (SD) time to TEE reporting was significantly improved with MTD data entry vs PC data entry [233 (676) min vs 1,103 $(3,830)$ min, respectively; mean difference 870 min; 95\% confidence interval (CI) 293 to 1,448; $P=0.003]$, and median (IQR) time was also significantly improved [46 (163) min vs 126 (1,000) min, respectively; median difference $80 \mathrm{~min} ; P=0.0002]$. The incidence of report delinquency with MTD data entry vs PC data entry was also significantly reduced $[2.1 \%$ vs $6.8 \%$, respectively; mean difference $2.2 \%$; $95 \%$ CI 0.5 to $9.0 ; P=0.02$ ].

Conclusion Implementation of a MTD system for data entry leads to improved TEE reporting time and reduces TEE reporting delinquency. Further studies are required to determine whether this strategy enhances quality of reporting, optimizes communication between care teams, and improves outcomes without increasing costs. 


\section{Résumé}

Objet La communication en temps opportun des constatations de l'échocardiographie transœsophagienne peropératoire (ETO) à l'équipe de soins postopératoires est essentielle pour optimiser les soins au patient. Nous avons comparé l'utilisation d'un système d'ordinateur personnel $(P C)$ à l'utilisation d'une tablette mobile (TM) pour la saisie des données d'ETO au point de service et nous avons émis l'hypothèse qu'un système basé sur les TM raccourcirait le délai de soumission du rapport préliminaire d'ETO et diminuerait de 50 \% l'incidence des rapports manquants.

Méthodes Dans cette étude de cohorte historique, nous avons passé en revue 508 rapports d'ETO périopératoires saisis par des anesthésistes cardiothoraciques. Les rapports ont été regroupés selon que les données avaient été saisies sur un PC (groupe PC) ou sur un système de TM (groupe TM). Le délai de fourniture du rapport d'ETO était défini par le temps écoulé entre la sortie du patient de la salle d'opération et le moment de la création du rapport. Les rapports étaient définis comme manquants s'ils étaient créés plus de $24 \mathrm{~h}$ après l'examen initial. Le délai d'émission du rapport d'ETO et l'incidence des rapports manquants ont été comparés entre les deux groupes.

Résultats Le délai moyen (É.T.) d'émission d'un rapport d'ETO a été significativement amélioré quand les données étaient saisies sur un système de TM comparativement à un PC (respectivement, 233 [676] min contre 1103 [3 830] min; différence moyenne $870 \mathrm{~min}$; intervalle de confiance à $95 \%$ [IC] : 293 à 1 448; $p=0,003$ ), et le délai médian (IQR) a été également significativement amélioré (respectivement, 46 [163] min contre 126 [1 000] min; différence médiane $80 \mathrm{~min} ; P=0,0002)$. L'incidence des rapports manquants avec la saisie des données sur TM par rapport à la saisie sur PC a été aussi significativement réduite (respectivement, 2,1\% contre 6,8\%; différence moyenne 2,2 \%; IC à $95 \%$ : 0,5 à 9,0; $P=0,02$ ).

Conclusion La mise en place d'un système de TM pour la saisie des données entraîne une amélioration des délais de soumission des comptes rendus d'ETO et diminue l'incidence des rapports manquants d'ETO. D'autres études sont nécessaires pour déterminer si cette stratégie améliore la qualité des rapports, optimise les communications entre les équipes de soins et améliore les résultats sans augmenter les coûts.

Transesophageal echocardiography (TEE) can have a direct impact on hemodynamic management and clinical decisionmaking in the intensive care unit (ICU), especially in the immediate postoperative period. ${ }^{1,2}$ The consequences of delayed TEE reporting include an increased risk of miscommunication during handoff to the postoperative care team and, accordingly, may impose a risk to patient safety. In addition, inadequate communication of pertinent TEE information may lead to implementation of inappropriate medical management strategies as well as the potential for increased cost through subsequent redundant echocardiography testing. ${ }^{3}$ Early TEE reporting provides documentation of TEE findings in the electronic medical record (EMR) shortly after the patient arrives in the ICU, ameliorating miscommunication during handoff. The American Society of Echocardiography (ASE) Continuous Quality Improvement Guidelines recommend that TEE providers generate a perioperative TEE report within $24 \mathrm{hr}$ of the exam. ${ }^{4}$

In order to enhance the quality of a TEE service within a system increasingly reliant on an EMR, it is imperative that new technology be afforded maximal leverage so as to improve efficiency in perioperative TEE reporting, improve communication, and potentially reduce unnecessary costs. Mobile tablet device (MTD) technology is increasingly being used as a clinical tool ${ }^{5,6}$ and has been shown to improve efficiency in electronic physician order entry systems. ${ }^{7}$ Nevertheless, academic institutions have not widely adopted MTDs for TEE reporting, and there is little evidence to support a benefit in using the MTD over traditional data entry for reporting TEE results. At our institution, a web-based data entry system is used to generate TEE reports. An overview of fellow workflow for TEE reporting overlapping with perioperative patient flow is described in the Figure. During a case, the fellow trainee assigned to the TEE service performs and reviews the TEE images with the assigned staff anesthesiologist. At our institution, the primary anesthesia team provides a verbal report of the TEE findings during ICU transfer, and additionally, fellow trainees enter TEE data into a TEE reporting website using a conventional personal computer (PC) workstation after completion of the cardiac surgical procedure. In our TEE service, approximately $10 \%$ of the reports are generated $>24 \mathrm{hr}$ after the procedure, and therefore are not in compliance with the ASE quality improvement guidelines. In an effort to improve timeliness of TEE reporting and compliance with ASE guidelines, we implemented a MTD-based point-of-care TEE data entry system. We hypothesized that using MTD-based point-of-care TEE data entry would reduce the overall time to generate a TEE report and reduce the number of delinquent reports (entered $>24 \mathrm{hr}$ after performance of the TEE examination) by $50 \%$.

\section{Methods}

This study was approved (March 4, 2013) by the Duke University Institutional Review Board.

All cardiothoracic anesthesia fellows were educated on the delinquent report rate and the consequences of inefficient reporting on the quality of care. The 


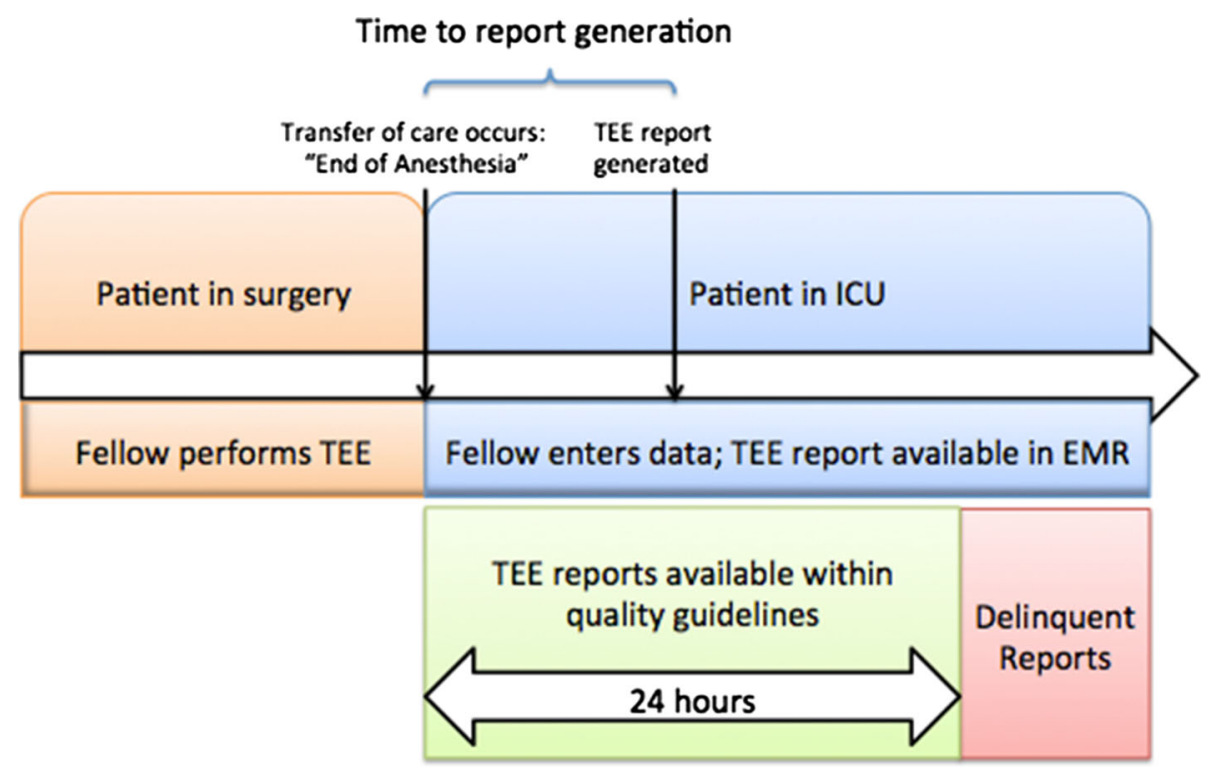

Figure Workflow for perioperative TEE reporting overlapping with the perioperative cardiac surgery patient flow. The TEE fellow is responsible for performing the perioperative TEE while the patient is in the OR, and for entering data for the report within $24 \mathrm{hr}$ of the exam. In this example, as the cardiothoracic surgical patient is transferred from the OR (orange) to the ICU (blue), the time from the "end of anesthesia care" until the TEE report is generated is measured as the "time to report generation". All reports entered after $24 \mathrm{hr}$ were defined as delinquent. TEE $=$ transesophageal echocardiogram; OR = operating room; EMR = electronic medical record; ICU $=$ intensive care unit

Time to TEE reporting was defined as the time from the "end of anesthesia" time stamp on the EMR until the time stamp indicating when the TEE report was generated (Figure). Reports that were generated prior to the "end of anesthesia" time were indicated with a negative value. Reports that were generated 24 hours after "end of anesthesia" time were considered delinquent.

Our a priori primary analysis was performed on the entire group, excluding those studies reported "after hours". In addition, we performed a subgroup analysis of those studies reported only "after hours". All cardiothoracic operations and TEEs performed during the evenings (7 pm -7 am) and weekends (Friday, $7 \mathrm{pm}$ Monday, $7 \mathrm{am}$ ) were considered in this "after hours" subgroup, as there is no fellow designated to perform and report TEEs during this time in the current system.

\section{Statistical analysis}

We assessed the difference in mean time to TEE reporting between the PC and MTD groups using a Student's $t$ test with unequal variances. We expected a large variability in times to generate reports due to variable clinical caseload and variable motivation of individual fellows to submit these reports in a timely fashion. The Wilcoxon signedrank test and median test were performed to assess the difference in distribution of the submitted times. A Chi 
square test was performed to compare the PC and MTD groups with regard to the percentage of delinquent reports. The Taylor series method was used to evaluate confidence intervals for the percentage of reports that were delinquent in the PC and MTD groups. A similar approach was used to analyze the subgroup of TEE reports during nights and weekends. The reported $P$ values for the $t$ test are twosided, with all other $P$ values being one-sided. We used SAS ${ }^{\circledR} 9.3$ (SAS Institute; Cary, NC, USA) for all analyses.

\section{Results}

During the study time periods, 508 perioperative TEE reports were performed, 235 in the PC group, entered by 13 individual fellows over eight weeks, and 273 in the MTD group, entered by a different group of 13 fellows over eight weeks. In the PC group, there were 176 weekday reports and 59 reports for nights and weekends. There were 195 weekday reports and 78 reports for nights and weekends in the MTD group. Analyses of the difference in TEE reporting times are summarized in the Table.

During the weekday, the median (IQR) time to TEE reporting for the MTD group was significantly shorter that for than the PC group [46 (163) min vs $126(1,000) \mathrm{min}$, respectively; median difference $80 \mathrm{~min} ; P=0.0002]$. The mean (SD) TEE reporting time was also significantly shorter for the MTD group than for the PC group [233 (676) $\min$ vs $1,103 \quad(3,830)$ min, respectively; mean difference $870 \mathrm{~min}$; $95 \%$ confidence interval (CI) 293 to $1,448 ; P=0.003]$. In the MTD group, the variance in mean reporting time differed significantly from that in the PC group $(P=0.002)$. We confirmed significantly different distributions of mean reporting time in the PC vs MTD group $(P=0.001)$.

During weekdays, $12(6.8 \%)$ of the 176 PC group reports were delinquent $v s$ only $4(2.1 \%)$ of the 195 MTD group reports. These delinquent reports resulted from six and two different fellows, respectively. Accordingly, after implementing the MTD program, the percentage of delinquent TEE reports decreased from $6.8 \%$ to $2.1 \%$ (mean difference $2.2 \%, 95 \%$ CI 0.5 to $9.0 ; P=0.02$ ).

\section{Subgroup analysis}

Analysis of night and weekend data showed that the mean (SD) time to TEE reporting may also have been significantly shorter in the MTD group $v s$ the PC group $[1,540(1,227) \min v s 4,784(11,830) \mathrm{min}$, respectively (mean difference $3,244 \mathrm{~min}$; 95\% CI 149 to 6,339; $P=0.040]$. The median time to generate reports was $894 \mathrm{~min}$ for the MTD group and 1,164 min for the PC group. The Wilcoxon test trended towards significantly different distribution in times between the PC and MTD groups $(P=0.05)$, while the median two-sample test did not show a difference $(P=0.18)$.

During nights and weekends, 26 of 59 reports (44.1\%) were delinquent in the PC group compared with 30 of 78 reports $(38.5 \%)$ in the MTD group, not significantly different (mean difference $8.5 \%$; $95 \%$ CI -11.3 to 22.5 ; $P=0.51)$.

\section{Discussion}

We confirmed our hypothesis that using MTD-based pointof-care TEE data entry, within the context of a fellow education program, reduced the overall time to generate TEE reports and, additionally, reduced the frequency of delinquent reports. This is a novel study reporting the value of using a MTD to improve efficiency within a perioperative TEE service. With increasing emphasis on accountability and high-quality patient care, new information technology solutions are constantly being

Table The differences in TEE reporting between the PC and MTD groups

\begin{tabular}{lllcc}
\hline & PC group & MTD group & Mean/Median Difference & $95 \%$ CI \\
\hline Weekdays $(n=371)$ & $(n=176)$ & $(n=195)$ & & $P$ value \\
Mean time (SD) [min] & $1,103(3,830)$ & $233(676)$ & 870 & 293 to 1,448 \\
Median time (IQR) [min] & $126(1,000)$ & $46(163)$ & 80 & 0.003 \\
Delinquent Reports & $6.8 \%$ & $2.1 \%$ & $2.2 \%$ & 0.0002 \\
Nights \& Weekends $(n=137)$ & $(n=59)$ & $(n=78)$ & & 0.02 \\
Mean time (SD) [min] & $4,784(11,830)$ & $1,540(1,227)$ & 3,244 & 0.0 \\
Median time (IQR) [min] & $1,164(2,739)$ & $894(1,909)$ & 270 & 0.04 \\
Delinquent Reports: & $44.1 \%$ & $38.5 \%$ & $8.5 \%$ & 0.18 \\
\hline
\end{tabular}

The table shows differences in mean and median times to generate TEE reports as well as differences in delinquent report rates during both weekdays and nights/weekends

$\mathrm{TEE}=$ transesophageal echocardiogram; $\mathrm{PC}=$ personal computer group; $\mathrm{MTD}=$ mobile tablet device group; $\mathrm{SD}=\mathrm{standard}$ deviation; $\mathrm{IQR}=$ interquartile range; $\mathrm{CI}=95 \%$ confidence interval 
introduced to enhance clinical efficiency. Mobile tablet devices and smartphones are routinely being used to access applications that help with health care decision-making. Devices such as MTDs introduce a novel way to bring information and education to the bedside or to send data rapidly to a centralized information resource. ${ }^{6-9}$ There is substantial evidence to support the use of handheld devices for access to information, data management, electronic documentation, ordering and prescribing, and decision support and education ${ }^{10-12}$ Nevertheless, despite the widespread availability of MTDs among health care providers, there is little evidence of their value in improving efficiency in the workplace. ${ }^{13}$

This paradigm is especially important when such transfer of information is used in critically ill patients. Typically, the first few hours in the intensive care unit (ICU) after cardiac surgery include a critical time of fluctuating hemodynamics, dynamic myocardial function, and bleeding that may be accompanied by rapid fluid shifts. Clinical decision-making in the ICU often hinges on information obtained from the TEE exam performed in the operating room. Importantly, this information must be available to care providers in a timely manner. It can be argued that, in a relatively unstable patient, any time lapse from the patient's arrival in the ICU to receipt of results is too long. Nevertheless, the clinical significance of the time lapse or the reduction in time to TEE reporting shown here is unknown. It is reasonable to expect that the availability of these results to the ICU team one hour and $20 \mathrm{~min}$ (median) earlier, as our study found, would assist with decision-making, potentially reducing or even eliminating the need for repeat echocardiographic assessment during this period.

The subgroup analysis of reports entered on nights and weekends revealed a delinquent reporting rate of approximately $40 \%$ in both groups. This was a somewhat expected finding, as the on-call team performs all perioperative TEEs during this period, but the data are not entered until the following working day. Unexpectedly though, there was a significant difference in mean time to TEE reporting between the PC and MTD groups during nights and weekends. The range was very large (Table), however, with a few extreme cases likely driving the nonsignificant results. It could be argued that, if MTD technology were the only factor making a difference, one would expect as large an impact in the evening/night and weekend cases as during weekdays. This can best be explained by the fact that our on-call duties are covered primarily by residents or fellows who are not expected or trained to enter TEE reports electronically. In addition, the MTDs are kept locked in the fellow reporting office after hours and are not available to residents. Alternative explanations for the difference might be the different personal motivations of the two different groups of fellows. Nevertheless, this difference was not achieved in the median time to generate reports or in the percent of delinquent reports (PC group $44.1 \%$ vs MTD group $38.5 \%$ ), likely due to our small sample size. Given the high percentage of delinquent reporting in this group, linked to both the on-call and TEE service structure, this matter has been identified as a future target for our internal continuous quality improvement in TEE reporting.

There are several limitations to this study - principally related to study design and data gathering. The study is retrospective in nature and therefore subject to both time and selection bias. There were 13 fellow trainees in each of the PC and MTD groups studying over two months in January and February 2012 and in January and February 2013, respectively. For our cardiothoracic anesthesia fellows, the TEE training is part of a one-year fellowship, and there is a learning curve in the first six months in terms of acclimating to the new hospital and performing and interpreting TEEs. Whereas fellows arrive to the program with varying amounts of TEE experience and training, by six months they have acclimated to the new system and can perform and interpret a basic perioperative TEE exam, which is why we chose our time periods for study. That is, we chose mid-year in training as our time period for study because fellows have been initiated in the TEE program, have acquired basic TEE skills, and have gained familiarity with the web-based reporting system. This design is also a limitation, however, in that there may be potential differences in the personal motivation among the different groups of trainees. This could be a driver of clinical efficiency that explains the differences we found between the two non-homogenous groups.

Nevertheless, we opted for this strategy rather than studying the same group before and after implementation of the MTD-based protocol. It would be extremely difficult to randomize trainees into two groups, with and without an MTD, and impossible to blind them to the time to data entry as they frequently interact during the day, thereby potentially invalidating the results.

An additional limitation is that the focus of our study was on quantitative factors indicative of clinical efficiency, and we did not review the quality of the TEE reports generated. It is possible that the quality of the TEE reports were sacrificed for reduced time to TEE reporting. Future studies could evaluate and compare the quality of TEE reports generated by the two data entry strategies. Finally, our intervention (MTD) group not only used the iPAD for data entry, but they also received additional education and e-mail reminders of the importance of timely reporting. We cannot exclude the possibility that the improvements in time to TEE reporting were due to the education the 
fellows received in addition to the introduction of the novel MTD-based data entry system. In their training, we introduced the concept and importance of timely reporting with reminders via didactic lectures and e-mail and faculty interaction for all fellows, including those in the PC group. Nevertheless, it is possible that the timing, quality, or effectiveness of our educational endeavours was novel, intrinsically different, or better received in the MTD group.

Problems experienced during technology implementation included loss of hardware and network connectivity problems. The hardware loss was largely due to missing chargers, resulting in eventual loss of power until the charger was replaced. We used the "Wi-Fi" feature on the MTD with our hospital's secure network to connect to the web-based data entry portal. This ensured the protection of patient information but mandated a secure password. If the password is not updated with a change in user, the connectivity to the network is interrupted and no web-based data entry is permitted. A few users encountered "Wi-Fi" loss of connectivity to our network in certain parts of the hospital as well. To assist, we enlisted two faculty members who were willing to troubleshoot and update the MTD as frequently as needed.

Since we have implemented MTD-based report generation as the new standard at our institution, we anticipate that shorter times to TEE reporting will continue. Future studies should also focus on sustaining this improvement with this intervention as well as reviewing the quality of reporting as a balance measure. We are enthusiastic that the implementation of the MTD for perioperative TEE reporting will make communication among providers more effective in the immediate postoperative period. We anticipate that rapid availability of the electronic report to the intensive care and surgical teams will help to avoid gaps in communication during handoff. Further studies are needed to assess whether this enhanced reporting efficiency improves communication, changes management decisions based on early information and thereby improves outcomes, or reduces cost by reducing the number of repeat echocardiograms performed during ICU admission.

In summary, we showed that use of a MTD within a dedicated perioperative TEE training program improved the efficiency of TEE reporting by reducing the time to TEE reporting. In addition, the frequency of delinquent reports was significantly reduced (by more than 50\%), thereby improving compliance with recommended guidelines for continuous quality improvement for a perioperative TEE service. Further studies are required to determine whether this strategy enhances the quality of reporting, optimizes communication, improves outcomes, and/or reduces cost.

Disclosures This work was funded in part by the Duke University Graduate Medical Education Innovation Fund. There are no other commercial or non-commercial affiliations or conflicts of interest.

\section{References}

1. Huttemann E. Transoesophageal echocardiography in critical care. Minerva Anestesiol 2006; 72: 891-913.

2. Guarracino $F$, Baldassarri $R$. Transesophageal echocardiography in the OR and ICU. Minerva Anestesiol 2009; 75: 518-29.

3. Douglas PS, Garcia MJ, Haines DE, et al. ACCF/ASE/AHA/ ASNC/HFSA/HRS/SCAI/SCCM/SCCT/SCMR 2011. Appropriate Use Criteria for Echocardiography. A Report of the American College of Cardiology Foundation Appropriate Use Criteria Task Force, American Society of Echocardiography, American Heart Association, American Society of Nuclear Cardiology, Heart Failure Society of America, Heart Rhythm Society, Society for Cardiovascular Angiography and Interventions, Society of Critical Care Medicine, Society of Cardiovascular Computed Tomography, and Society for Cardiovascular Magnetic Resonance Endorsed by the American College of Chest Physicians. J Am Coll Cardiol 2011; 57: 112666.

4. Mathew JP, Glas K, Troianos CA, et al. ASE/SCA recommendations and guidelines for continuous quality improvement in perioperative echocardiography. Anesth Analg 2006; 103: 1416-25.

5. Tanaka PP, Hawrylyshyn KA, Macario A. Use of tablet (iPad(R)) as a tool for teaching anesthesiology in an orthopedic rotation. Rev Bras Anestesiol 2012; 62: 214-22.

6. Franko OI, Tirrell TF. Smartphone app use among medical providers in ACGME training programs. J Med Syst 2012; 36: 3135-9.

7. Patel BK, Chapman CG, Luo N, Woodruff JN, Arora VM. Impact of mobile tablet computers on internal medicine resident efficiency. Arch Intern Med 2012; 172: 436-8.

8. Panughpath $S G$, Kalyanpur A. Radiology and the mobile device: radiology in motion. Indian J Radiol Imaging 2012; 22: 246-50.

9. George P, Dumenco L, Dollase R, Taylor JS, Wald HS, Reis SP. Introducing technology into medical education: two pilot studies. Patient Educ Couns 2013; 93: 522-4.

10. Kuziemsky $C E$, Laul $F$, Leung $R C$. A review on diffusion of personal digital assistants in healthcare. J Med Sys 2005; 29: 33542.

11. Lindquist AM, Johansson PE, Petersson GI, Saveman BI, Nilsson $G C$. The use of the Personal Digital Assistant (PDA) among personnel and students in health care: a review. J Med Internet Res 2008; 10: e31.

12. Martins HM, Jones MR. What explains doctors' usage of mobile information and communication technologies? A comparison of US and Portuguese hospitals. AMIA Annu Symp Proc 2005; 2005: 495-9.

13. Prgomet M, Georgiou A, Westbrook JI. The impact of mobile handheld technology on hospital physicians' work practices and patient care: a systematic review. J Am Med Inform Assoc 2009; 16: 792-801. 